\title{
BMJ Open A double-blind randomised placebo- controlled trial of melatonin as an adjuvant agent in induction of labour (MILO): a study protocol
}

\author{
Kamala Swarnamani, ${ }^{1}$ Miranda Davies-Tuck (D) , ${ }^{1,2}$ Euan Wallace, ${ }^{2}$ Ben W Mol, ${ }^{2,3}$ \\ Joanne Mockler ${ }^{2,3}$
}

To cite: Swarnamani K, DaviesTuck M, Wallace E, et al. A double-blind randomised placebo-controlled trial of melatonin as an adjuvant agent in induction of labour (MILO): a study protocol. BMJ Open 2020;10:e032480. doi:10.1136/ bmjopen-2019-032480

- Prepublication history and additional material for this paper are available online. To view these files, please visit the journal online (http://dx.doi. org/10.1136/bmjopen-2019032480).

Received 20 June 2019 Revised 14 January 2020 Accepted 14 January 2020

Dheck for updates

(C) Author(s) (or their employer(s)) 2020. Re-use permitted under CC BY-NC. No commercial re-use. See rights and permissions. Published by BMJ.

${ }^{1}$ The Ritchie Centre, Hudson Institute of Medical Research, Clayton, Victoria, Australia ${ }^{2}$ Department of Obstetrics and Gynaecology, Monash University, Clayton, Victoria, Australia

${ }^{3}$ Monash Health, Clayton,

Victoria, Australia

Correspondence to

Dr Miranda Davies-Tuck;

Miranda.davies@hudson.org.au

\section{ABSTRACT}

Introduction Induction of labour (IOL) is a common practice. In Australia, up to $40 \%$ of women undergoing labour induction will ultimately have a caesarean section. As a biological role for melatonin in the onset and progress of labour has been demonstrated, we aim to test the hypothesis that addition of melatonin will reduce the need for caesarean section.

Methods and analysis This is a double-blind, randomised, placebo-controlled trial in women undergoing IOL at term. We plan to randomise 722 women (1:1 ratio) to receive either melatonin (four doses of $10 \mathrm{mg}$ melatonin: first dose - in the evening at the time of cervical balloon or Dinoprostone $\mathrm{PGE}_{2}$ vaginal pessary insertion, second dose-at time of oxytocin infusion commencement, third dose- 6 hours after the second dose, fourth dose-6 hours after the third dose) or placebo (same dosing regime). Participants who are having artificial rupture of the membranes only as the primary means of labour induction will receive up to three doses of the trial intervention. The primary outcome measure will be the requirement for a caesarean section. Secondary outcomes will include duration of each stage of labour and time from induction to birth, total dose of oxytocin administration, epidural rate, indication for caesarean section, rate of instrumental deliveries, birth within 24 hours of induction commencement, estimated blood loss, Apgar score at 5 min, neonatal intensive care unit admissions and participant satisfaction. Maternal melatonin levels will be measured immediately before commencement of the oxytocin intravenous infusion and 3 hours after and at the time of birth in order to determine any differences between the two trial arms.

Ethics and dissemination The study is conducted in accordance with the conditions of Monash Health HREC (RES-17-0000-168A). Findings from the trial will be disseminated through peer-reviewed publications and conference presentations.

Protocol version V.7.0, 30 July 2019.

Trial registration number ACTRN12616000311459, Universal trial number: (UTN) U1111-1195-3515.

\section{INTRODUCTION}

Induction of labour (IOL) is one of the most common obstetric interventions. In Australia,
Strengths and limitations of the study

- This is the first randomised placebo-controlled trial designed to study the effect of melatonin in reducing caesarean section rates.

- Both the participants and the clinicians providing care will be blinded to the trial intervention allocation, thus decisions regarding the need for a caesarean section cannot be influenced by the allocation.

- A potential limitation of this study is that it is conducted at a single health service.

the labours of $33 \%$ of women are induced, up from $25 \%$ only a decade earlier. ${ }^{12}$ IOL is principally performed with the intent of reducing risks to the mother and/or baby by simply calling an end to the pregnancy. Ideally, the induced labour progresses to a vaginal birth. However, despite randomised controlled trials indicating that IOL does not increase caesarean section rates $^{34}$ outside of the tight confines of trials, the reality for women in high-income nations is that up to $\sim 40 \%$ of women having their labours induced will require a caesarean section, this is particularly true if they are nulliparous. ${ }^{5-9}$

Failure of IOL resulting in a caesarean section is important for a number of reasons. First, it may be associated with disappointment for the woman. There are well-established links between the need for a caesarean birth and the risk of postnatal depression ${ }^{10}$ and post-traumatic stress disorder, particularly in first-time mothers. ${ }^{11}$ Women who require a caesarean section are also less likely to breastfeed their baby. ${ }^{12} 13$ An intrapartum caesarean is associated with greater maternal morbidity than vaginal birth, including higher rates of postpartum haemorrhage, endometritis, venous thromboembolism, a longer recovery time and an increased rate of hospital readmission. ${ }^{1415}$ Having a caesarean section is also 
associated with an increased risk of morbidities in subsequent pregnancies including preterm birth, abnormal placentation and uterine rupture. ${ }^{16} 17$ Improving the success of IOL is therefore important not only for the current labour but also for all future pregnancies.

It is most common practice in Australia for the IOL process to begin in the morning. Optimum staffing levels are considered the main driver for this. ${ }^{18}$ However, such timing may not be optimal for the woman. First formally reported over 60 years ago was the observation that spontaneous labour most commonly starts at night. ${ }^{19}$ In a study of 19000 labours, the peak time of onset was between 02:00 and 03:00. ${ }^{19}$ More interestingly, labours commencing between 23:00 and 01:00 were significantly shorter than those that commenced between 04:00 and 19:00. ${ }^{19}$ Over the years, these intriguing observations have been confirmed by others (reviewed in Serón-Ferré et $a l^{20}$ ). The accepted explanation underlying the observations has been that uterine muscle (myometrial) fibres are more sensitive to the effects of endogenous oxytocin at night. ${ }^{21}{ }^{22}$ The reason for this increased sensitivity at night is thought to be due to melatonin.

Melatonin (5-methoxy- $N$-acetyltryptamine) is an endogenous hormone produced primarily by the pineal gland. It provides circadian and seasonal timing cues. ${ }^{23} \mathrm{In}$ adults, melatonin levels remain low throughout the day. In the early evening, the levels begin to increase, peaking between 02:00 and 03:00 and then falling back down to low daylight concentrations again in the morning. ${ }^{24}$ This circadian rhythm of melatonin is amplified in pregnancy. In particular, healthy pregnant women have higher concentrations of melatonin both at night and during the day compared with non-pregnant women ${ }^{25}$ most likely due to de novo placental synthesis. ${ }^{26}$ In addition, maternal melatonin levels increase with advancing gestation peaking during labour and then falling rapidly after birth. ${ }^{26}$ The myometrium (uterine muscle) expresses the melatonin receptor MT2 and it is more highly expressed in labouring myometrium, collected at intrapartum caesarean section, than in myometrium from non-labouring women. ${ }^{27}$ The role of melatonin in the onset of active labour has not been widely studied; however, studies demonstrating increased myometrial expression of melatonin receptions among preterm birth suggest a role. ${ }^{26}$ Melatonin also increases the sensitivity of the myometrium to oxytocin-induced contractions. ${ }^{28}$ Cotreatment of an immortalised myometrial cell line with melatonin and oxytocin resulted in a twofold increase in contractile response compared with oxytocin alone. ${ }^{28}$ In these same studies, melatonin was also shown to increase the expression of the protein connexin 43, a gap-junction protein necessary for myometrial cell communication and the synchronisation of uterine contractions. ${ }^{28}$ Taken together, these in vivo and in vitro observations suggest that melatonin plays a biological role in the timing of onset of spontaneous labour and in the effectiveness of spontaneous uterine contractions in labour. As IOL with an oxytocin intravenous infusion normally occurs during the daytime in the Australian setting, women do not experience the physiological increases in melatonin that occur prior to going into spontaneous labour at night, we hypothesise that administering melatonin at the time the induction process commences will reduce the IOL failure rate. We will undertake a double blind, randomised, placebo-controlled trial of oral melatonin administration at IOL to reduce caesarean section rates in healthy women with a singleton pregnancy at full term.

\section{METHODS AND ANALYSIS}

This protocol has been designed in accordance with the SPIRIT (Standard Protocol Items: Recommendations for Interventional Trials) 2013 Guidelines (see online supplementary file 1, WHO Trial Registration Dataset).

\section{Study design}

This is a phase III double-blind, randomised, placebocontrolled trial.

\section{Trial timeline}

The study is conducted on March 2019-March 2022.

\section{Study setting}

Participants will be recruited at Monash Health, Monash Medical Centre, a level six university affiliated teaching hospital in metropolitan Melbourne, which provides care for up to 9000 women a year. Approximately 10 women who meet our inclusion and exclusion criteria are induced per week at this institution.

\section{Subjects}

Women with a singleton pregnancy who are undergoing IOL participated in the study.

\section{Inclusion criteria}

- $\geq 18$ years and $<50$ years of age.

- Nulliparous or multiparous (para 1-3) women with a singleton pregnancy in cephalic presentation.

- Full term ( $\geq 37$ weeks).

- Bishop's score $<5$ (a strongest predictor of failure of induction). ${ }^{29}$

- Intact membranes.

- Method of induction includes the use of a cervical balloon catheter and/or Dinoprostone $\mathrm{PGE}_{2}$ (gel/ tablets/pessary) or women who are only having an amniotomy as the initial method of IOL.

- No known significant maternal or obstetric medical condition that would affect melatonin pharmacokinetics or maternal safety.

Specific exclusion criteria

- Fetal growth restriction $<10$ th centile with Doppler changes.

- Any known congenital anomaly of the fetus.

- Any known abnormal karyotype of the fetus.

- Non-reassuring fetal status. 
- Not willing to or inability to follow the procedures outlined in participant information and consent form.

- Known allergy or sensitivity to melatonin and its formulation.

- Mentally or legally incapacitated or not able to provide informed consent.

- Participation in another trial where there is pharmaceutical or any other nutritional intervention.

\section{Participant recruitment and informed consent}

Potential participants are those who are booked for labour induction from across the hospital's maternity departments. A researcher, not involved in the provision of clinical care, will approach all eligible women and provide the Participant Information and Consent Form (see online supplementary file 2). The researcher will then provide a verbal explanation of the trial, including a description of the trial processes, the voluntary nature of the trial and that a decision to participate, or not, will not affect their clinical care. The woman will be encouraged to discuss their participation with others of their choosing and given sufficient time to consider if they wish to take part, or not. If the woman agrees to participate, she will provide written informed consent.

\section{Randomisation and blinding}

A perinatal epidemiologist with no involvement in the clinical trial will prepare the randomisation sequence that is given to an onsite dedicated clinical trial pharmacy. Randomisation will occur on a 1:1 ratio of melatonin to placebo. Both the melatonin and the placebo tablets will be indistinguishable and contained in individually preprepared bottles by the clinical trial pharmacist who is likewise not involved in participant recruitment of trial conduct. Neither the researcher, the clinical staff nor the participants will know whether melatonin or placebo tablets have been administered. At the time of recruitment, each participant will be deidentified and assigned a unique trial code. Subsequently, all data and tissue samples collected from the participant will be labelled and stored only with this associated code.

\section{Study intervention}

Participants will be given either melatonin $10 \mathrm{mg}$ (Circadin prolonged release melatonin, manufactured by Aspen Pharma, New South Wales, Australia) tablets or visually identical placebo tablets manufactured by a Good Manufacturing Practice (GMP)-compliant compounding pharmacy. The administration regimen of the trial intervention will be determined by the method of labour induction.

\section{Cervical balloon and/or Dinoprostone $\mathrm{PGE}_{2}$ (pessary/tablet/gel) (evening admission) \\ Participants who are having a cervical balloon and/or Dinoprostone $\mathrm{PGE}_{2}$ (pessary/tablet/gel) will receive up to four doses of the trial intervention: first dose-in the evening at the time of initial cervical balloon or Dinopro- stone $\mathrm{PGE}_{2}$ vaginal pessary/gel/tablet insertion, second}

dose-at the time of the oxytocin intravenous infusion commencement, third dose-6hours after the second dose and fourth dose -6 hours after the third dose.

Artificial rupture of membranes only (morning admission)

Participants who are having artificial rupture of the membranes (ARM) only as the primary means of labour induction will receive up to three doses of the trial intervention: first dose-following the ARM procedure-at the time of the oxytocin infusion commencement, second dose -6 hours after the first dose and third dose- 6 hours after the second dose.

For both of the trial intervention administration regimens, if birth occurs before all of the doses have been administered, no further tablets will be given.

\section{Outcomes}

Primary outcome measure

Caesarean section.

\section{Secondary outcome measures}

1. Total length of labour (including the duration of first, second, third stages of labour).

2. Induction commencement to birth interval (time of start of induction (either with balloon or cervidil or ARM and oxytocin) to the time of delivery).

3. Total dose of oxytocin administered.

4. Estimated blood loss in millilitres (from birth to 24 hours postpartum).

5. Instrumental vaginal birth (total and within 24 hours of IOL commencement).

6. Uterine tachysystole (more than five contractions in 10 min without fetal heart rate changes).

7. Apgar score at $5 \mathrm{~min}$.

8. Participant satisfaction with the labour assessed via a five-point Likert scale (How would rate your overall experience of your induction?)

9. Postnatal length of stay.

10. Admission of the baby to neonatal intensive care unit (ICU) (from birth to discharge of the baby) as per the decision of consultant neonatologist.

11. Participant melatonin levels will be determined prior to the administration of the second dose of the trial intervention and commencement of the oxytocin intravenous infusion, 3 hours after the administration of the second dose of the trial intention and within 20 min of giving birth.

12. Umbilical cord blood melatonin levels (collected at the time of birth).

13. Economic impact: The total resources and costs will be compared from the time of admission to the discharge of the participant and her baby. Economic outcome measures include personnel costs, medication and other clinical costs; this will be performed in compliance with the International Society for Pharmacoeconomics and Outcomes Research good practice standards. ${ }^{30}$ 


\section{Covariates/Confounders}

Maternal age (years), maternal weight and height at time of induction, parity, maternal self-reported country of birth, Bishop score/cervical dilation at time of labour onset, pain relief in labour, time of birth and indication for operative birth, if relevant.

\section{Sample and data collection}

Participant blood samples are collected: (1) just prior to commencement of the intravenous oxytocin infusion and intervention (tablet) administration, (2) 3 hours after the oxytocin infusion has started and (3) at birth. Melatonin concentration will be measured using a commercial kit (RK-MEL2; Buhlmann Laboratories, Schonenbuch, Switzerland). Prior to participant discharge from hospital, all data relevant to the outcomes (including safety outcomes) will be collected by accessing their hospital records. The participants will be visited in the postnatal ward by a member of the research team to ask for general feedback regarding their participation in the trial.

\section{Confidentiality and data collection}

Confidentiality of all the participants data will be strictly maintained by all the researchers and in line with the national and local guidelines. All members of the Data Safety Monitoring Board (DSMB) will be provided with deidentified data only (ie, bearing only the unique participant code). All data collected from the participants will be gathered on a standardised case report form and entered onto a password-protected dedicated trial database. The original hard copy, case report form will be stored in a locked filling cabinet located in a secure, departmental location. After completion of the trial, all trial-related data and records will be retained for a minimum of 15 years in accordance with Monash Health and National Health Medical Research Council (NHMRC) requirements for clinical trials. After this period has been reached, all trial-related data and records will be deleted or undergo secure destruction.

\section{Sample size}

This trial has been powered to detect a $10 \%$ absolute reduction in the rate of the primary outcome: caesarean section. This was determined to reflect a clinically significant decrease in caesarean section rate as per the healthy people 2020 targets, ${ }^{31}$ an initiative of the American Federal Government that recommends a $10 \%$ reduction in caesarean rates. The rate of caesarean section at term among primiparous women undergoing IOL at our health service from 2009 to 2015 is $\sim 37 \%$. Therefore, to detect a reduction in caesarean section rates from $37 \%$ to $27 \%$, we will recruit a total of 722 women (361 in each arm). With a total of 722 women, the detectable differences in each of our secondary outcomes are detailed in table 1 .

\section{Statistical analysis}

All analyses will be performed using intention-to-treat. All continuous data will be assessed for normality. Characteristics of the two groups will be tabulated and compared using the appropriate statistical test $\left(\chi^{2}\right.$, independent t-test, Mann-Whitney test). Differences in the primary outcome will be assessed by logistic regression adjusting for confounders/baseline imbalances between groups if appropriate. Differences in the secondary outcomes will be assessed using the appropriate regression method adjusting for confounders/baseline imbalances between groups if appropriate. Rates of the primary and secondary outcomes will also be reported by parity. The costs will be calculated separately for each mother/infant pair. A $\mathrm{p}$ value of $<0.05$ will be considered to indicate statistical significance. Analyses will be undertaken using StataCorp V.12 (StataCorp. 2011. Stata Statistical Software: Release 12. College Station, Texas, USA: StataCorp LP.)

\section{Adverse events}

Adverse events and serious adverse events will be identified according to International Conference on Harmonisation Good Clinical Practice (ICH GCP) definitions.

Table 1 Detectable differences in secondary outcomes

\begin{tabular}{lcl}
\hline Secondary outcomes & $\begin{array}{l}\text { Mean (SD) or proportion in untreated } \\
\text { group based on our health service data }\end{array}$ & $\begin{array}{l}\text { Two-sided detectable difference based } \\
\text { on primary outcome sample size }\end{array}$ \\
\hline Length of first stage of labour (hours) & $8.22(5.66)$ & -1.2 or 1.2 hours \\
\hline Length of second stage of labour (min) & $55(65)$ & -13.6 to 13.6 min \\
\hline $\begin{array}{l}\text { Length of third stage of labour (min) } \\
\text { Duration of oxytocin infusion (hours) }\end{array}$ & $6(13.6)$ & -2.8 to 2.8 \\
\hline $\begin{array}{l}\text { Total volume of oxytocin administered } \\
\text { (milliunits/min) }\end{array}$ & $69.6(43)$ & -0.93 or 0.93 hours \\
Blood loss $(\mathrm{mL})$ & $459.8(328)$ & -8.9 or 8.9 units \\
\hline Apgar score $<7$ at 5 min & $2 \%$ & -68.5 or $68.5 \mathrm{~mL}$ \\
\hline Admission to NICU & $1.3 \%$ & $0.0 \%$ or $6.1 \%$ \\
\hline Epidural use & $31 \%$ & $0.0 \%$ or $4.9 \%$ \\
\hline
\end{tabular}

NICU, neonatal intensive care unit. 
The lead principal investigator (PI) who is an AHPRA registered medical doctor is responsible for all trialrelated medical decisions; this includes the assessment unexpected events and, where necessary, attribution of causality to those which have been determined as adverse events.

To date, clinical studies have not demonstrated any serious adverse reactions to the use of melatonin. However, there may be unexpected reactions associated with melatonin when used at IOL in pregnant women that we do not yet know about. Therefore, participants and their babies will be assessed for any adverse events from the time of induction commencement until their discharge from hospital signifying their completion in the trial. All adverse events will be recorded and reported in accordance with the requirements of the Sponsor, Monash Health and NHMRC (National Health and Medical Research Council) guidance. The responsibility for the assessment, documentation and reporting of all such events is held by the PI.

For the purposes of this clinical trial, it is reasonable to remain mindful of the potential for side effects and adverse reactions. The accompanying literature and/or product information for melatonin shows that adverse effects are rare, that is, likely to occur in fewer than 1 in 1000 persons which includes: Irritability, nervousness, restlessness insomnia, abnormal dreams, anxiety, migraine, lethargy, psychomotor hyperactivity (restlessness associated with increased activity), dizziness, somnolence (tiredness), high blood pressure, (upper) abdominal pain, indigestion, mouth ulceration, dry mouth, hyperbilirubinaemia (changes in the composition of your blood which could cause yellowing of the skin or eyes (jaundice), inflammation of the skin (dermatitis, night sweats, pruritus (itching), rash, dry skin, pain in extremities, menopausal symptoms, asthenia (feeling of weakness), chest pain, excretion of glucose in urine, excess proteins in the urine, abnormal liver function and weight increase, shingles, reduced number of white blood cells in the blood, decreased number of platelets in the blood, high level of fatty molecules in the blood, severe chest pain due to angina, feeling your heartbeat (palpitations,. low serum calcium levels in the blood, low sodium levels in the blood, altered mood, aggression, agitation, crying, stress symptoms, disorientation, early morning awakening, increased sex drive, depressed mood, depression, loss of consciousness or fainting, memory impairment, disturbance in attention, dreamy state, restless legs syndrome, poor quality sleep, 'pins and needles' feeling (paraesthesia) reduced visual acuity (visual impairment), blurred vision, watery eyes, dizziness when standing or sitting, vertigo, hot flushes, gastro-oesophageal reflux, gastrointestinal disorder, blistering in the mouth, tongue laceration, gastrointestinal upset, vomiting, abnormal bowel sounds, flatulence (wind), salivary hypersecretion (excess saliva production), halitosis (bad breath), abdominal discomfort, gastric disorder, inflammation of the stomach lining, eczema, erythema (skin rash), hand dermatitis, psoriasis, pruritic rash (itchy rash), nail disorder, arthritis, muscle spasms, neck pain, night cramps, tiredness, pain, thirst, passing large volumes or urine, presence of red blood cells in the urine, urination during the night, increased liver enzymes, abnormal blood electrolytes and abnormal laboratory tests.

\section{Data and safety monitoring board}

As the use of melatonin is not indicated in current use in pregnancy for IOL, a DSMB was established. The DSMB comprises two Australian Health Professional Regulatory Agency (AHPRA) registered medical doctors, one a neonatologist and one obstetrician, as well as a biostatistician. Data will be provided to DSMB in a deidentified form following the recruitment of $30 \%$ and $60 \%$ participants, or at other times as requested by the DSMB or the Sponsor, Monash Health Research Directorate and Human Research Ethics Committee. No interim statistical analysis of the primary or secondary outcomes is planned. The DSMB are advised to advocate complete cessation, or re-evaluation, of the trial conduct, if it is evident that either arm of the trial is associated with a statistically significant increase in or a $50 \%$ increased rate above the baseline ratio of any, or all, of the following outcome:

- Episodes of any or all of the following: uterine tachysystole (more than five contractions in $10 \mathrm{~min}$ without fetal heart rate changes), uterine hypertonus (defined as contractions lasting longer than $2 \mathrm{~min}$ in duration or occurring within $60 \mathrm{~s}$ of each other without fetal heart rate abnormalities) and uterine hyperstimulation(tachysystole or uterine hypertonus with fetal heart rate abnormalities).

- Estimated blood loss $\geq 500 \mathrm{~mL}$ for vaginal birth or $\geq 750 \mathrm{~mL}$ at caesarean birth.

- Participant entry to ICU, high dependency unit or coronary care unit.

- Uterine rupture.

- Apgar score $<7$ at $5 \mathrm{~min}$ of age requiring active resuscitation (this include oxygen therapy, intermittent positive pressure ventilation, endotracheal intubation, continuous positive airway pressure or laryngoscopy). - Maternal or perinatal death.

\section{Trial modification and discontinuation}

There will be no allowances to modify the trial intervention administration regimen or other trial-related processes. The participant maybe or discontinued from the trial for reasons including not able to follow the procedures, in violation with the protocol, adverse drug reaction to the trial tablets or at their own request. The PI will determine if more participants will be needed to increase the sample size to compensate for loss to follow-up/discontinuation/withdrawal. The trial may also be discontinued at the request of the Human Research Ethics Committee/ Research Directorate or at the request of the DSMB. 


\section{Unblinding}

Deliberate unblinding in the trial may occur in the following circumstances:

- To make clinical treatment decisions or when an unexpected serious adverse event occurs and the intervention must be made known.

- At the request of the DSMB.

- At the conclusion of the study to determine the effect of the intervention.

For individual unblinding, the lead clinical PI will assess and authorise the need for unblinding. The process for individual unblinding will involve the clinical PI contacting the site pharmacy and providing the participant identification number. On unblinding, the participant will be recorded as withdrawn from the trial.

\section{Patient and public involvement}

There has been no patient or public involvement in the trial design. All women will be asked about their satisfaction with the IOL (secondary outcome measure) and feedback regarding their participation in the trial will also be sought.

\section{Ethics and dissemination}

The clinical trial will be carried out in accordance with the conditions of Monash Health Human Research Ethics Committee (HREC approval), National Health Medical Research Council (NHMRC) National Statement on Ethical Conduct in Human Research 2007 (updated 2018) and the NHMRC Australian Code for the Responsible Conduct of Research (2018). ${ }^{32} 33$ On completion of the trial, its findings will be presented at scientific meetings and published in peer-reviewed journals.

\section{DISCUSSION}

The rates of IOL are increasing globally. The ultimate aim of IOL, beyond a healthy mother and baby, is to achieve a vaginal delivery. However, for up to $37 \%$ women having their labour induced, this does not occur and they will require a caesarean section. The trial is the first doubleblind, randomised, placebo-controlled trial of melatonin administration at IOL. If successful, it will show that this inexpensive and safe supplement will improve the rate of vaginal birth and reduce total healthcare costs. As such, this trial could be responsible for the first fundamentally new development in labour care for many decades

\section{Trial status}

The trial commenced March 2019.

\section{Protocol amendments}

The PIs will be responsible for informing the sponsor, Monash Health, of any proposed protocol amendments, in accordance with local requirements.

\section{Dissemination policy}

Following completion of the trial, it is intended that the aggregated results will be published in peer-reviewed journals and presented at scientific conferences. A summary of the trial findings will be provided to those participants who have requested to receive this.

Twitter Miranda Davies-Tuck @DaviesTuck and Euan Wallace @euan_wallace

Contributors JM, MD-T and EW conceptualised the trial. JM, MD-T, EW and BWM designed the trial and wrote the trial protocol. KS drafted the protocol manuscript for publication. All authors provided contributions to the editing and approval of the final manuscript.

Funding This trial is funded by a National Health and Medical Research Council (NHMRC) Project grant (APP1123498).

Competing interests None declared.

Patient consent for publication Not required.

Provenance and peer review Not commissioned; externally peer reviewed.

Open access This is an open access article distributed in accordance with the Creative Commons Attribution Non Commercial (CC BY-NC 4.0) license, which permits others to distribute, remix, adapt, build upon this work non-commercially, and license their derivative works on different terms, provided the original work is properly cited, appropriate credit is given, any changes made indicated, and the use is non-commercial. See: http://creativecommons.org/licenses/by-nc/4.0/.

ORCID iD

Miranda Davies-Tuck http://orcid.org/0000-0003-1918-5538

\section{REFERENCES}

1 Australian Institute of Health and Welfare. Australia's mothers and babies 2017-in brief. In: Perinatal statistics series. Canberra: AlHQ, 2019.

2 Laws PJ, Li Z, Sullivan EA. Australia's mothers and babies 2008. Perinatal statistics series. Canberra: AlHW, 2010.

3 Grobman WA, Rice MM, Reddy UM, et al. Labor induction versus expectant management in low-risk nulliparous women. $N$ Engl J Med 2018;379:513-23.

4 Walker KF, Bugg GJ, Macpherson M, et al. Randomized trial of labor induction in women 35 years of age or older. $N$ Engl J Med 2016;374:813-22.

5 Zhang J-W, Branch W, Hoffman M, et al. In which groups of pregnant women can the caesarean delivery rate likely be reduced safely in the USA? A multicentre cross-sectional study. BMJ Open 2018;8:e021670.

6 Hunt R, Davey M, Anil S, et al. Victorian perinatal services performance indicators report 2016-17. Melbourne: Safer Care Victoria, Victorian Government, 2018.

7 Nippita TA, Trevena J, Patterson J, et al. Variation in inter-hospital rates of induction of labour. BJOG 2015;122:354-5.

8 Lee YYC, Roberts CL, Patterson JA, et al. Unexplained variation in hospital caesarean section rates. Med J Aust 2013;199:348-53.

9 Walsh JM, Hehir MP, Robson MS, et al. Mode of delivery and outcomes by birth weight among spontaneous and induced singleton cephalic nulliparous labors. Int J Gynaecol Obstet 2015;129:22-5.

10 Biaggi A, Conroy S, Pawlby S, et al. Identifying the women at risk of antenatal anxiety and depression: a systematic review. J Affect Disord 2016;191:62-77.

11 Clout D, Brown R, Sociodemographic BR. Sociodemographic, pregnancy, obstetric, and postnatal predictors of postpartum stress, anxiety and depression in new mothers. J Affect Disord 2015;188:60-7.

12 Patel A, Bucher S, Pusdekar Y, et al. Rates and determinants of early initiation of breastfeeding and exclusive breast feeding at 42 days postnatal in six low and middle-income countries: a prospective cohort study. Reprod Health 2015;12(Suppl 2):S10.

13 Portela DS, Vieira TO, Matos SM, et al. Maternal obesity, environmental factors, cesarean delivery and breastfeeding as determinants of overweight and obesity in children: results from a cohort. BMC Pregnancy Childbirth 2015;15:94.

14 Hammad IA, Chauhan SP, Magann EF, et al. Peripartum complications with cesarean delivery: a review of Maternal-Fetal medicine units network publications. J Matern Fetal Neonatal Med 2014;27:463-74.

15 Clark EAS, Silver RM. Long-term maternal morbidity associated with repeat cesarean delivery. Am J Obstet Gynecol 2011;205:S2-10. 
16 Silver RM, Landon MB, Rouse DJ, et al. Maternal morbidity associated with multiple repeat cesarean deliveries. Obstet Gynecol 2006;107:1226-32.

17 Cong A, de Vries B, Ludlow J. Does previous caesarean section at full dilatation increase the likelihood of subsequent spontaneous preterm birth? Aust N Z J Obstet Gynaecol 2018;58:267-73.

18 Thorsell M, Lyrenäs S, Andolf E, et al. Starting time for induction of labor and the risk for night-time delivery. Sex Reprod Healthc 2011;2:113-7.

19 Charles E. The hour of birth; a study of the distribution of times of onset of labour and of delivery throughout the 24-hour period. $\mathrm{Br} J$ Prev Soc Med 1953;7:43-59.

20 Serón-Ferré M, Ducsay CA, Valenzuela GJ. Circadian rhythms during pregnancy. Endocr Rev 1993;14:594-609.

21 Honnebier MB, Nathanielsz PW. Primate parturition and the role of the maternal circadian system. Eur J Obstet Gynecol Reprod Biol 1994;55:193-203.

22 Zahn V, Hattensperger W. [Circadian rhythm of pregnancy contractions]. Z Geburtshilfe Perinatol 1993;197:1-10.

23 Slominski RM, Reiter RJ, Schlabritz-Loutsevitch N, et al. Melatonin membrane receptors in peripheral tissues: distribution and functions. Mol Cell Endocrinol 2012;351:152-66.

24 Claustrat B, Brun J, Chazot G. The basic physiology and pathophysiology of melatonin. Sleep Med Rev 2005;9:11-24.
25 Nakamura Y, Tamura H, Kashida S, et al. Changes of serum melatonin level and its relationship to feto-placental unit during pregnancy. J Pineal Res 2001;30:29-33.

26 Sagrillo-Fagundes L, Soliman A, Vaillancourt C. Maternal and placental melatonin: actions and implication for successful pregnancies. Minerva Ginecol 2014;66:251-66.

27 Olcese J, Lozier S, Paradise C. Melatonin and the circadian timing of human parturition. Reprod Sci 2013;20:168-74.

28 Sharkey JT, Puttaramu R, Word RA, et al. Melatonin synergizes with oxytocin to enhance contractility of human myometrial smooth muscle cells. J Clin Endocrinol Metab 2009;94:421-7.

29 Grobman WA. Predictors of induction success. Semin Perinatol 2012;36:344-7.

30 McGhan WF, Al M, Doshi JA, et al. The ISPOR good practices for quality improvement of cost-effectiveness research Task force report. Value Health 2009;12:1086-99.

31 U.S. Department of Health and Human Services Office of Disease Prevention and Health Promotion. Healthy people 2020, 2014.

32 NHMRC. The National statement on ethical conduct in human research 2007 (updated 2018. National Health and Medical Research Council, 2018.

33 NHMRC. Australian code for the responsible conduct of research National Health and Medical Research Council, 2018. 